\title{
Recommendations based on International Guidelines for Safe Dental Practice During Covid-19
}

\author{
Dr. Ashutosh Kumar Singh, ${ }^{1}$ Dr. Manoj Humagain, ${ }^{2}$ Dr. Nikita Khanal ${ }^{3}$ \\ ${ }^{1}$ Department of Oral \& Maxillofacial Surgery, College of Medical Sciences, Bharatpur, Nepal \\ ${ }^{2}$ Department of Periodontics, Kathmandu University School of Medical Sciences, Dhulikhel, Nepal \\ ${ }^{3}$ Bharatpur Samudayik Hospital Bharatpur, Nepal
}

\begin{abstract}
The shutdown of dental practices after the announcement of COVID-19 has been implemented worldwide. Aerosol generating procedures and face to face contact with the patients put dental professionals at highest risk of disease transmission. There is a looming risk of increasing severity of dental conditions and burden on treatment providers if required dental treatments are kept on hold for an indefinite time. There is a need for definite guidelines to safely resume dental practice in Nepal, based on international experiences during COVID-19 pandemic and international dental societies recommendations. We have compiled multiple recommendations and guidelines from reputed international organizations and collected evidence from dental professional's work from China and Italy during COVID-19 crisis and attempted to put forward some recommendations and guidelines that can be followed for a safe dental practice in Nepal.

Keywords: Clinical guidelines; COVID-19; dentistry.
\end{abstract}

\section{INTRODUCTION}

The novel coronavirus was initially named 2019-nCoV and officially as severe acute respiratory syndrome coronavirus 2 (SARS- CoV-2). ${ }^{1}$ It was first identified in December 2019 in Wuhan, the capital of China's Hubei province. On January 30, 2020, the World Health Organization (WHO) announced that this outbreak had constituted a public health emergency of international concern. There are currently 15,491 PCR positive cases in Nepal, out of which 6415 have recovered and 34 are dead.

Due to the inherent nature of dental procedures causing splatters, fomites and aerosols the risk of cross-infection may be high between dental practitioners and patients and between consecutive patients as well as dental auxiliary staff. For private dental clinics and hospitals in a country like ours that are affected with COVID-19, strict and effective infection control protocols are urgently needed. This article, based on evidence and relevant guidelines and research, introduces the essential knowledge about COVID-19 and nosocomial infection in dental settings and

\section{Correspondence: \\ Dr. Ashutosh Kumar Singh, \\ Department of Oral \& Maxillofacial surgery \\ College of Medical Sciences, Bharatpur, Nepal \\ Email: drashutoshkumarsingh@gmail.com \\ Citation}

Singh AK, Humagain M, Khanal N. Recommendations based on International Guidelines for Safe Dental Practice During Covid-19. J Nepal Soc Perio Oral Implantol. 2020;4(7):22-30

DOI: https://doi.org/10.3126/jnspoi.v4i1.30900 provides recommended management protocols for dental practitioners and specialists.

\section{Clinical history and transmission characteristics}

Patients with symptomatic COVID-19 have been the main source of transmission, recent evidence suggests that asymptomatic patients and patients in their incubation period are also carriers of SARS-CoV-2. ${ }^{2}$ This epidemiologic feature of COVID-19 poses the greatest challenge to control its transmission as it is difficult to identify and quarantine these patients in time, which can result in community spread of SARS-CoV-2. ${ }^{3}$ The incubation period of COVID-19 has been estimated at 5 to 6 days on average to as long as 14 days, which is now the commonly adopted duration for medical observation and quarantine of (potentially) exposed persons. ${ }^{2,4}$

Current observations suggest that people of all ages are generally susceptible to this new infectious disease but people with a debilitating illness, chronic comorbidities and old age are more susceptible to severe illness requiring hospitalization and critical care. However, those who are in close contact with patients with symptomatic and asymptomatic COVID-19, including health care workers and other patients in the hospital, are at higher risk of SARSCoV-2 infection. The common presentation is of high fever and dry cough, while some also present with shortness of breath, fatigue, and other atypical symptoms, such as muscle pain, confusion, headache, sore throat, diarrhoea, and vomiting. 5,6

The diagnosis of COVID-19 can be based on a combination 
of epidemiologic information (e.g., a history of travel to or residence in affected region 14 days prior to symptom onset), clinical symptoms, CT imaging findings, and laboratory tests (e.g., reverse transcriptase-polymerase chain reaction [RT-PCR] tests on respiratory tract specimens) according to standards of the WHO (2020a). ${ }^{7}$ It should be very clear to clinicians that a single negative RT-PCR test result from suspected patients does not exclude infection and clinically, we should be alert of patients with an epidemiologic history of travel to and from clusters and restricted zones, COVID-19-related symptoms, contact history with suspected or confirmed patients and/or positive CT imaging results. ${ }^{8-12}$ So far, there is no level I evidence from randomised controlled trials to recommend any specific anti-nCoV treatment, so the management of COVID-19 still is largely supportive (WHO 2020a). ${ }^{7}$ Currently, the approach to manage COVID-19 is to control the source of infection; use infection prevention and control measures to lower the risk of transmission; and provide early diagnosis, isolation, and supportive care for affected patients. ${ }^{13}$ A variety of drugs and interventions are being investigated that are potentially more effective and curative (e.g., lopinavir, remdesivir). Development of vaccine and mass vaccination is the only long term solution to this pandemic but vaccine development requires at least 18 months of development and trial phases to be deemed safe for mass clinical application. ${ }^{13-18}$ The dental staff and dental practitioners are considered to be at highest risk of acquiring the infection because of prolonged face to face exposure to patients, exposure to respiratory secretions and aerosols produced during aerosol-generating procedures like ultrasonic scaling and cavity/access preparation using a high-speed air rotors with water jet cooling systems. ${ }^{19}$ Evidence suggests that aerosols produced during dental procedures, procedures that cause the patient to cough can propel a high viral load in the procedure room and contaminate various surfaces in the room where the virus can remain active for up to
9 days. $^{20}$ Recently there were observations made that prove the salivary glands act as the reservoir for the virus and active and live virus was isolated from the saliva of patients who were asymptomatic or were considered free of the disease. ${ }^{21}$ Salivary gland reserve and saliva has been proposed as the major route of transmission in few recent studies which also suggest saliva as a better sample for COVID-19 testing compared to respiratory secretions. The expression of ACE2 in minor salivary glands was higher than that in lungs (lung medium PTM [transcripts per kilobase of exon model per Million mapped reads] $=1.010$, minor salivary gland medium PTM $=2.013$ ), which suggests salivary glands could be a potential target for COVID-19. The positive rate of COVID-19 in patients' saliva can reach $91.7 \%$, and saliva samples can also cultivate the live virus. This suggests that COVID-19 transmitted by asymptomatic infection may originate from infected saliva. ${ }^{19,21}$

Dental procedures stimulate salivation and cough thus increasing the risk of fomites carrying viral particles that remain airborne for at least 20 minutes in the air and up to 9 days on surrounding solid surfaces like glass, metal and plastics, thus enabling cross-transmission through the air and contact transmission in a dental clinic. ${ }^{20,22}$ Dental treatment is characterised by relatively long operation times, which results in the persistent existence of aerosols within a large area of the clinical office and introduces a potential risk of spreading disease and at the same time, patients have no protection during the treatment process. ${ }^{23}$ Use of sharp and pointed instruments can also pose a puncture risk and clogged handpieces and suction with backflow from carrier patients can put successive patients at risk of transmission. ${ }^{24}$ The incubation period and the possibility of patients concealing medical history could easily lead to the spread of disease. Lack of strict supervision and rampant quackery based dental services in our country, could become hotspots for disease transmission in the community.

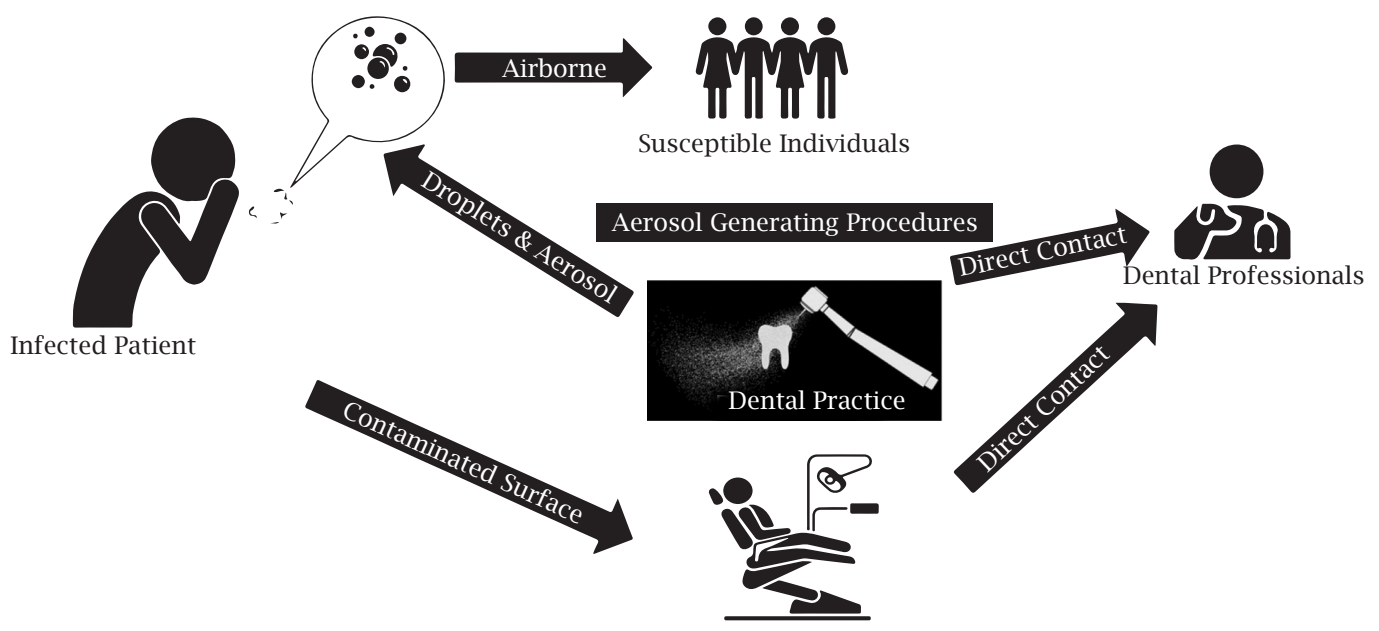

Figure 1: Transmission routes in dentistry.

Adapted and changed from Peng, X., Xu, X., Li, Y. et al. Transmission routes of 2019-nCoV and controls in dental practice. Int J Oral Sci. 2020:12, 9. https://doi.org/10.1038/s41368-020-0075-9 
Based on these facts we can imagine the highest level of infection control and personal protection that will be required for dental procedures to be performed safely in the foreseeable future until a vaccine or treatment becomes commonplace. ${ }^{25}$ Though there is still no clear consensus on continuation, resumption and extent of dental practice in coming days of this pandemic, based on the risk of cross-transmission and high risk to dental professionals, most countries and dental communities were advised to stop general dental procedures and only provide essential emergency dental care which has been followed in our country too. The complete shutdown of dental treatment will reduce the risk of transmission cluster from dental offices but in long term will lead to accumulation of cases and conversion of an easily treatable case into an emergency or non-salvageable case. ${ }^{26}$ Relapse of orthodontics cases and reinfection of under treatment root canal cases can occur. This case burden will strain the dental community in the near future, therefore, resumption of dental treatments with very clear case prioritisation, infection control, transmission reduction and staff safety protocols are the need of the day. ${ }^{27}$ As the graph of COVID-19 infection lowers down and flattens we should be ready to resume our services with universal standards of care based on multiple evidence and international guidelines tailored to suit our national resource availability and community characteristics.

\section{Objectives of the guidelines}

According to the US Government COVID-19 response plan published by the US Department of Health and Human Services (HHS) on 13 March 2020, this COVID- 19 pandemic could last over 18 months. ${ }^{28}$

Closing dental practices during the pandemic can reduce the number of affected individuals but will increase the suffering of the individuals in need of urgent dental care. This lack of guidelines can, on one hand, increase the nosocomial COVID-19 spread through dental health care facilities, and on the other hand, deprive patients' in need of the required urgent dental care.

It is the purpose of this work to develop a guideline for dental patients' management during and after the COVID-19 pandemic. These guidelines will safeguard dental care professionals from acquiring COVID-19 infections and prevent cross-transmission among patients.

1. Screening every asymptomatic patient meticulously.

2. Isolation and transfer of suspected patients to the local health authority for testing and keeping contact of every patient for contact tracing if required.

3. Considering every patient as a potential asymptomatic COVID-19 carrier.

4. Considering recently recovered patients as potential virus carriers for at least 30 days after the recovery confirmation by a laboratory test.

5. Identifying the urgent need of the patient and focusing on managing it with minimally invasive procedures.

6. Categorising dental treatment according to the urgency of the required treatment and the risk and benefit associated with each treatment.

7. Identifying the required dental treatment for each patient and the risks and benefits associated with that treatment.

8. Using contact and airborne precautions including proper aerosol-generating procedures personal protective equipment (PPE) for every procedure.

9. Preparing clinic/hospitals/institutions with COVID-19 safety standards.

10. Preparation and training of reception staff, dental hygienists, assistants, and cleaners for maintaining safe practice.

11. Preparing checklist-based PPE donning and doffing protocol based on the level of exposure and procedure.

12. Preparation of operatory and maintenance of high standards of disinfection in the operatory.

13. Protocols for instrument sterilization and waste management from Dental settings.

14. Accidental exposure management recommendations.

15. Optimization of resources like PPE, Facemasks, respirators, and Eyewear during scarcity.

\section{Recommendations for dental practice}

The safety recommendations from different international health agencies can be practically summarised into a few salient common features:

1. Identification of suspected COVID-19 positive cases with a history questionnaire and clinical examination at the initial contact point with a contactless thermometer. ${ }^{29}$ The screening questionnaire has to be modified to include recent contact with anyone with travel history to cluster areas and clinical history of immediate family members.

2. A possible case of COVID-19 needs to meet both the clinical symptoms AND have a travel history, including travel to, or transit through (for any length of time), the identified risk countries OR contact with a confirmed case of coronavirus.

3. If a patient is presenting with symptoms after 14 days, they do not meet the case definition and can be handled as normal.

4. Advice, analgesia and antimicrobials should be the 
primary care for the majority of patients and only acute problems should be provided face to face treatments.

5. Isolation of suspected patients in a specifically prepared isolation room or area, and protocol preparation for handing over such patients to the regional or local COVID-19 medical team for further management.

6. Preparation of waiting area and receiving area with standard distancing guidelines, airflow and respiratory transmission safety protocol. ${ }^{30}$

7. Preparation of dental office and procedure area with reduction of all non-essential surfaces and decorations.

8. Minimisation of clinic staff with a rotation duty roster and minimising accompanying persons with strict protocol in place for only single accompanying person with one patient.

9. Preparation of special and dedicated procedure areas with clearly demarcated potential transmission zones.

10. Provision of universal and special safety precautions in place with checklists before, during and after patient contact.

11. Availability of personal protective equipment for all the staff and treatment providers based on grading of transmission risk.

12. Strict disinfection of procedure area according to international standards between patients to prevent cross-transmission utilising locally available and effective resource like $0.1 \%$ Hypo or chlorine as surface disinfectant. ${ }^{22}$

13. Wipe and disinfect the object surfaces as well as the floor with a disinfectant containing 2,000 mg/L of effective chlorine at least twice a day, and disinfect as soon as possible if there is contamination. Pay special attention to high-frequency contact surfaces (such as various handles, buttons, instrument panels, stair walkways, etc. ${ }^{22}$

14. Once the oral treatment is completed, each chair used by the patient should be wiped with $75 \%$ ethanol or 2,000 mg/L disinfectant containing effective chlorine.

15. Daily screening logs for staff and immediate voluntary quarantine if suspected for COVID- 19 infection.

These administrative recommendations are advised to reduce the risk of cross-transmission among dental staff and patients as well as among successive patients. Absolute minimisation of potentially contaminable surfaces and strict disinfection protocol will eradicate the possibility of contact transmission. Provision of a dedicated procedure area will limit the potential contamination and will be easier to disinfect regularly. Identification and clinical suspicion of potential asymptomatic carriers should be of paramount importance and for that, a printed questionnaire with positive and negative response has been used universally. ${ }^{28,31,32}$ Once the administrative controls are in place, next comes the reduction of risk to dental professionals and staff who come in contact with patients. There are some universal recommendations based on evidence that can guide the personal safety measures required in dental office. ${ }^{33}$

1. Availability of soap, antimicrobial hand washes and sinks for proper handwash technique according to WHO protocol. ${ }^{34}$

2. Availability of alcohol-based hand sanitizers with $>70 \%$ alcohol concentration in the receiving area, procedure area and waiting for area. ${ }^{30}$

3. Preparation of patients with antimicrobial oral rinse (0.5\% hydrogen peroxide or $1 \%$ povidone-iodine solution) to reduce viral load in respiratory and oral secretions. Traditionally used chlorhexidine based oral rinse are least effective against novel coronavirus and should never be used..$^{35}$

4. Universal precautions with appropriate facemask, protective gown, eyewear, full face shield, head and shoe covers and staff specially trained to properly don and doff the PPE and check for the fit of facemask. ${ }^{36}$

5. Intraoral x-ray examination is the most common radiographic technique in dental imaging; however, it can stimulate saliva secretion and cough. Therefore, extraoral dental radiographs, such as panoramic radiography and cone-beam $\mathrm{CT}$, are appropriate alternatives during the outbreak of COVID-19. ${ }^{37}$

6. For the treatment of patients with trauma or inflammation without aerosol generation, healthcare workers should use enhanced grade-2 protection which includes surgical mask, isolation gown, head cap, eye googles, disposable shoe covers and face shield (non-aerosol generation operations in Figure 1). When using high-speed turbo-charging handpieces and oral ultrasonic scalers that can generate aerosols and create an immersion environment, dental workers should use enhanced grade-3 protection which includes medical coveralls and filtering facemask respirators like N95 mask in addition to the grade 2 protection mentioned for non-AGP (aerosol generation operations in Figure 1).

7. Remove the protective mask/goggles and protective clothing when leaving the clinic; perform personal hygiene after work and have situational awareness about what your hands touch. Frequent handwashing and use of alcohol-based hand sanitizer is absolutely a must. ${ }^{38}$ 
Installation and Use of HEPA air filters in procedure room."

If multiple chairs are to be used, they are kept at 6 feet distance.

If possible separated by a physical barrier like a screen.
Use of waterless and motor-driven handpieces as a replacement for air-driven high-speed water-cooled handpieces.

Use of extraoral suction apparatus with barrier mechanism and performing the dental procedure under indirect vision. Similar clear barriers and suction devices are currently being used by Ent specialists and Anaesthesiologists for procedures that pose the risk of exposure to respiratory and oral secretions.

Special handpieces with anti-retraction suction and backflow preventive mechanism can reduce the risk of clogging and harbouring microbes causing the risk of cross-transmission.
Chemo mechanical cavity and access preparation and minimization of handpiece usage.

Pulp exposure could be made with chemo mechanical caries removal under rubber dam isolation and a high-volume saliva ejector after local anaesthesia; then, pulp devitalization can be performed to reduce the pain.

Minimal use of ultrasonic scalers for periodontal procedures and maximising the use of aerosol freehand instruments.

Figure 2: Engineering protocols for safety measures of protection in dental offices, dental chair and while operating patients.

Last but not the least, once the administrative protocol is in place to identify and isolate suspected cases, and respiratory or contact transmission risk is reduced by personal safety measures, there still is potential for some asymptomatic carrier to cross all safety barriers and reach the dental chair for treatment. Some engineering recommendation to reduce aerosol generation and reduction of exposure to potential viral load in such cases is the final frontier of protection in dental offices (Figure 2). ${ }^{33,39}$ Use of rubber dam if available and training to apply and use the rubber dam with high vacuum suction apparatus. There is a definite need for concerned authorities like national dental association, medical council and universities to take a lead and train all the stakeholders as well as supervise, monitor and evaluate periodically. It is also a professional, moral and ethical responsibility of dental health care providers to avail such
Optimizing the availability of Filtering facepiece respirators (FFRs)

\section{Optimizing the al-ailability of PPE}

Maximize the use of engineering controls, such as barriers and maintained ventilation systems.

Maximize the use of administrative controls, such as altering work practices to minimize patient contacts. Use reusable PPE that can be reprocessed.

Use PPE beyond the manufacturerdesignated shelf life for training.

Consider allowing DHCP to extend the use of respirators, facemasks, and eye protection, beyond a single patient contact by thorough decontamination process.

Consider using intact PPE that is beyond the manufacturer-designated shelf life for patient care activities. Carefully prioritize PPE use for selected care activities.

This could include reserving sterile gowns and gloves for urgent sterile patient procedures, such as surgery, and reserving respirators for aerosolgenerating procedures.

If no commercial PPE is available, carefully consider if alternative approaches will reduce the risk of staff exposure and are safe for patient care.
Optimizing the availability a evewear and fice shields

Fully chy (air dty or use clean absorbent towels).

While wearing gloves. carefully wipe the inside. followed by the outside of the face shield or goggles using a clean cloth saturated with neutral detergent solution or cleaner wipe.

Wipe the outside of face shield or goggles with clean water or alcohol to remove msidue.

Carefully wipe the outside of the face shield or goggles using a wipe or clean cloth saturated with a hospital disinfectant solution.
If FFR decontamination is considered. these methods do not appear to break down filtration or compromise the FFR. UV chambers are widely available in all denta practices and can be used for decontamination and reuse if a shortage exists.

Figure 3: Optimizing the availability of Filtering facepiece respirators (FFRs), PPE, eyewear and face shields. ${ }^{43-50}$ 
1. Recommendations for dental professionals with high risk performing aerosol generating procedures or nonaerosol-generating procedure on asymptomatic suspected or confirmed COVID-19 patient.

- Stop all health care interaction with patients for 14 days after the last day of exposure to a confirmed COVID-19 patient

- $\quad$ Be tested for COVID-19 virus infection and quarantine for 14 days in a designated setting.

2. Recommendations for dental professionals with low risk performing non-aerosol procedures or procedure on asymptomatic and non-confirmed cases.

- $\quad$ Reinforce contact and droplet precautions when caring for all patients with acute respiratory illness and standard precautions to take care of all patients.

- $\quad$ Reinforce airborne precautions for aerosol-generating to all suspects and confirmed COVID-19 patients.

- $\quad$ Reinforce the rational, correct and consistent use of personal protective equipment when exposed to confirmed COVID-19 patients. Apply WHO's "My 5 Moments for Hand Hygiene" before touching a patient, before any clean or aseptic procedure, after exposure to body fluid, after touching a patient, and after touching patient's surroundings and Practice respiratory etiquette at all times. ${ }^{50-54}$

\section{Figure 4: Management protocol in case of accidental exposure. ${ }^{5-62}$}

training and keep themselves updated in the new normal way of dental practice.

Our health system is facing a crisis state due to lack of PPE, facemasks, respirators, eyewear and face shields. In such a crisis state some modified protocol for decontamination and reuse of PPE have been advised (Figure 3). ${ }^{40,41}$ Scientific evidence-based decontamination and reuse of essential protective equipment is need of the day and these suggestions can be adapted till there are resumption and availability of protective equipment (Summary of recommendations suited to conditions in Nepal are provided in Table 1). ${ }^{38}$ This is the new normal for routine dental practice in near future and we have to learn and adapt to the situation sooner than later. $^{42}$

It is necessary to wear gloves during any contact and always perform hand hygiene frequently. Strict adherence to recommendation based on evidence and guidelines will help to decide regarding when to resume dental services, how to prioritise cases, and how to effectively prevent crosstransmission among the providers, staff and patients. ${ }^{40,41,51}$ Knowledge of basic infection control measures, technical safety barriers, evidence-based personal safety measures and discipline are the best protective personal equipment for this pandemic. ${ }^{52-54}$ We cannot decline or postpone dental treatments for an indefinite period so sooner than later we have to be prepared to resume our services safely and scientifically. ${ }^{55,56}$

\section{Accidental Exposure management}

Despite following all the safety precautions, some staff might get exposed to a confirmed case of Coronavirus during treatment and in such cases, exposure protocol should be in place to prevent cross-transmission (Figure 4). ${ }^{56,57}$

Table 1: Summary of recommendations for safe dental practice during COVID-19 in a low GDP country like Nepal while optimizing the availability of resources. ${ }^{30}$

Prior to dental treatment (patients at home)

Phone triage questionnaire

Organization of patient flux

Prior to dental treatment (patients entering the practice)

Body temperature measurement

Hand hygiene (patient)

Waiting room

Environmental disinfection

Nonclinical staff clothing
Provide limitations to dental office access. Book appointments to avoid crowding of patients No accompanying persons if possible. If not possible, allow just one and ask the person to wait outside in the waiting hall.

Assess potential presence of fever via contactless thermometer

Use of hand sanitizers for hand disinfection when entering the dental office

Provide adequate ventilation with simple table fans and open windows

Removal of all unnecessary furniture and decorations that could favour cross-infection

Avoid long stay in the waiting room.

Avoid the simultaneous presence of $>2$ patients

Respect the distance of in between patients

Use of $0.1 \%$ sodium hypochlorite or $70 \%$ isopropyl alcohol for the disinfection of all surfaces

Application of face masks (filtering facepiece level 2 or 3), goggles and face shields with simple UV sterilization in dental UV chamber. 


\begin{tabular}{|c|c|}
\hline $\begin{array}{l}\text { Preparation to dental treatment (dentist and patient) } \\
\text { Patient preparation } \\
\text { Clinical staff hand washing } \\
\text { Clinical staff clothing }\end{array}$ & $\begin{array}{l}\text { Use of disposable shoe covers } \\
1 \text {-min mouth rinse with } 0.2 \% \text { to } 1 \% \text { povidone } \\
\text { Hand washing for at least } 60 \text { s and then } 60 \% \text { hydroalcoholic } \\
\text { solution application prior to wearing gloves } \\
\text { Application of face masks (filtering facepiece level } 2 \text { or } \\
\text { 3), shields, surgical glasses, long- sleeved water- resistant } \\
\text { gown, surgical cap, shoe cover. } \\
\text { Simple OT gowns can be used if commercial full body PPE } \\
\text { not available. }\end{array}$ \\
\hline $\begin{array}{l}\text { Dental treatment } \\
\text { Instruments Surfaces } \\
\text { Minimizing aerosol production }\end{array}$ & $\begin{array}{l}\text { Preparation of all instruments in advance. Fix number of } \\
\text { instrument trays and give appointments accordingly. } \\
\text { Total protection through disposable covers or wipe sur- } \\
\text { faces with disinfectant and provide a gap of minimum } 20 \\
\text { mins between patients. } \\
\text { Avoid, when possible, use of handpieces/ultrasonic instru- } \\
\text { ments } \\
\text { Use of rubber dam, if available } \\
\text { High speed surgical aspiration system } \\
\text { If possible, prefer } 4 \text { - hands technique } \\
\text { Limit overall treatment time if possible }\end{array}$ \\
\hline $\begin{array}{l}\text { After dental treatment Ventilation } \\
\text { Instruments } \\
\text { Personal Protection } \\
\text { Hand Hygiene (dentist) }\end{array}$ & $\begin{array}{l}20 \text { min air change strongly advised } \\
\text { Removal of disposable protection from the surfaces } \\
\text { Disinfection of shields and glasses with } 70 \% \text { isopropyl } \\
\text { alcohol Hand washing for at least } 60 \text { s and then } 60 \% \\
\text { hydroalcoholic solution application. }\end{array}$ \\
\hline
\end{tabular}

Conflict of interest: None.

\section{REFERENCES}

1. Wu Y, Ho W, Huang Y, Jin DY, Li S, Liu SL, et al. SARS-CoV-2 is an appropriate name for the new coronavirus. Lancet. 2020;21:949-50.

2. Cheng ZJ, Shan J. 2019 Novel coronavirus: where we are and what we know. Infection. 2020;48(2):155-63.

3. Rothan HA, Byrareddy SN. The epidemiology and pathogenesis of coronavirus disease (COVID-19) outbreak. J Autoimmun. $2020 ; 109: 102433$.

4. World Health Organization. Coronavirus Disease 2019 (COVID-19): Situation Report [Internet].WHO, Geneva. Available from: https://apps. who.int/iris/handle/10665/331686.

5. Tong ZD, Tang A, Li KF, Li P, Wang HL, Yi JP, et al. Zhang YL, Yan JB. Potential Presymptomatic Transmission of SARS-CoV-2, Zhejiang Province, China, 2020. Emerg Infect Dis. 2020;26(5):1052-4.

6. Zhai P, Ding Y, Wu X, Long J, Zhong Y, Li Y. The epidemiology, diagnosis and treatment of COVID-19. Int J Antimicrob Agents. 2020;55(5):105955.

7. World Health Organization. Clinical Management of COVID-19: Interim Guidance [Internet].WHO, Geneva.

8. Rothe C, Schunk M, Sothmann P, Bretzel G, Froeschl G, Wallrauch C,et al. Transmission of 2019-nCoV Infection from an Asymptomatic Contact in Germany. N Engl J Med. 2020;382(10):970-1.

9. Udugama B, Kadhiresan P, Kozlowski HN, Malekjahani A, Osborne M, Li VYC, et al. Diagnosing COVID-19: The Disease and Tools for Detection. ACS Nano. 2020;14(4):3822-35.

10. Koyama T, Weeraratne D, Snowdon JL, Parida L. Emergence of Drift Variants That May Affect COVID-19 Vaccine Development and Antibody Treatment. Pathogens. 2020;9(5):324.

11. Mustafa Z, Ghaffari M. What Do We Need to Know to Improve Diagnostic Testing Methods for the 2019 Novel Coronavirus? Cureus. 2020;12(5):e8263.

12. Bachelet VC. Do we know the diagnostic properties of the tests used in COVID-19? A rapid review of recently published literature. Medwave. 2020;20(3):e7890.

13. Guo YR, Cao QD, Hong ZS, Tan YY, Chen SD, Jin HJ, et al. The origin, transmission and clinical therapies on coronavirus disease 2019 (COVID-19) outbreak - an update on the status. Mil Med Res. 2020;7(1):11.

14. Nicola M, O'Neill N, Sohrabi C, Khan M, Agha M, Agha R. Evidence based management guideline for the COVID-19 pandemic - Review article. Int J Surg. 2020;77:206-16. 
15. Sanders JM, Monogue ML, Jodlowski TZ, Cutrell JB. Pharmacologic Treatments for Coronavirus Disease 2019 (COVID-19): A Review. JAMA. 2020; 2020;323(18):1824-1836.

16. Chen WH, Strych U, Hotez PJ, Bottazzi ME. The SARS-CoV-2 Vaccine Pipeline: an Overview. Curr Trop Med Rep. 2020:1-4.

17. Lurie N, Saville M, Hatchett R, Halton J. Developing Covid-19 Vaccines at Pandemic Speed. N Engl J Med. 2020;382(21):1969-73.

18. Thanh Le T, Andreadakis Z, Kumar A, Gómez Román R, Tollefsen S, Saville M, et al. The COVID-19 vaccine development landscape. Nat Rev Drug Discov. 2020;19(5):305-6.

19. Peng X, Xu X, Li Y, Cheng L, Zhou X, Ren B. Transmission routes of 2019-nCoV and controls in dental practice. Int J Oral Sci. 2020;12(1):9

20. Van Doremalen N, Bushmaker T, Morris DH, Holbrook MG, Gamble A, Williamson BN, et al. Aerosol and Surface Stability of SARS-CoV-2 as Compared with SARS-CoV-1. N Engl J Med. 2020;382(16):1564-7.

21. Sri Santosh T, Parmar R, Anand H, Srikanth K, Saritha M. A Review of Salivary Diagnostics and Its Potential Implication in Detection of Covid-19. Cureus. 2020;12(4):e7708.

22. Kampf G, Todt D, Pfaender S, Steinmann E. Persistence of coronaviruses on inanimate surfaces and their inactivation with biocidal agents. J Hosp Infect. 2020;104(3):246-51.

23. Ge ZY, Yang LM, Xia JJ, Fu XH, Zhang YZ. Possible aerosol transmission of COVID-19 and special precautions in dentistry. Version 2. J Zhejiang Univ Sci B. 2020;21(5):361-8.

24. D'Cruz L. PPE or not PPE - that is the question. Br Dent J. 2020;228(10):753-4.

25. Coulthard P. Dentistry and coronavirus (COVID-19) - moral decision-making. Br Dent J. 2020;228(7):503-5.

26. Dziedzic A, Wojtyczka R. The impact of coronavirus infectious disease 19 (COVID-19) on oral health. Oral Dis. 2020:10.1111/odi.13359. [published online ahead of print, 2020 Apr 18].

27. Alharbi A, Alharbi S, Alqaidi S. Guidelines for dental care provision during the COVID-19 pandemic. Version 2. Saudi Dent J. 2020;32(4):1816. [published online ahead of print, 2020 Apr 7].

28. American Nurses Association. Coronavirus Disease (COVID-19). Am Nurses Credentialing Cent. 2020;(March 2020):1-7. https://www. nursingworld.org/practice-policy/work-environment/health-safety/disaster-preparedness/coronavirus/.

29. American Dental Association. Patient Screening Form [Internet].ADA. Available from: https://success.ada.org/ /media/CPS/Files/Open\%20 Files/ADA_Return_to_Work_Toolkit.pdf

30. Arrive BP. Coronavirus Disease 2019 ( COVID-19 ) Get Your Clinic Ready for Coronavirus Disease 2019 help protect your patients and healthcare workers from COVID-. 2019;2019:2019-2020.

31. Centers for Disease Conttrol and Prevention. Get Your Clinic Ready for Coronavirus Disease 2019 (COVID-19). [Internet].CDC. Available from: https://www.cdc.gov/coronavirus/2019-ncov/hcp/clinic-preparedness.html

32. Dental S. Drugs for the Management of Dental Problems During COVID-19 Pandemic. Scottish Dent Clin Eff Progr. 2020.

33. Hua CG, Liu ZQ, Wang Q, Yang Z, Xu QH, Zhang J. [Strategy of dental clinics to cope with the epidemic period of infectious diseases based on the experience of corona virus disease outbreak]. Hua Xi Kou Qiang Yi Xue Za Zhi. 2020;38(2):117-21.

34. American Dental Association. Hand Hygiene for the Dental Team [Internet].ADA. Available from: https://success.ada.org/en/practicemanagement/staff/hand-hygiene-for-the-dental-team

35. Zhang W, Jiang X. Measures and suggestions for the prevention and control of the novel coronavirus in dental institutions. Front Oral Maxillofac Med. 2020;2:4.

36. Centers for Disease Conttrol and Prevention. Strategies for Optimizing the Supply of Isolation Gowns [Internet].CDC. Available from: https://www.cdc.gov/coronavirus/2019-ncov/hcp/ppe-strategy/isolation-gowns.html

37. Wang Y, Zhou CC, Shu R, Zou J. [Oral Health Management of Children during the Epidemic Period of Coronavirus Disease 2019]. Sichuan Da Xue Xue Bao Yi Xue Ban. 2020;51(2):151-4.

38. Centers for Disease Conttrol and Prevention. Strategies for Optimizing the Supply of Eye Protection [Internet].CDC. Available from: https:// www.cdc.gov/coronavirus/2019-ncov/hcp/ppe-strategy/eye-protection.html

39. Medical Advisory Secretariat. Air cleaning technologies: an evidence-based analysis. Ont Health Technol Assess Ser. 2005;5(17):1-52.

40. Khan MM, Parab SR. Simple Economical Solution for Personal Protection Equipment (Face Mask/Shield) for Health Care Staff During COVID 19. Indian J Otolaryngol Head Neck Surg. 2020:1-5.

41. Khan MM, Parab SR. Safety Guidelines for Sterility of Face Shields During COVID 19 Pandemic. Indian J Otolaryngol Head Neck Surg. 2020:1-2.

42. Proffitt E. What will be the new normal for the dental industry? Br Dent J. 2020;228(9):678-80.

43. Umer F, Haji Z, Zafar K. Role of respirators in controlling the spread of novel coronavirus (COVID-19) amongst dental healthcare providers: a review. Int Endod J. 2020;53(8):1062-7.

44. Viscusi DJ, Bergman MS, Eimer BC, Shaffer RE. Evaluation of five decontamination methods for filtering facepiece respirators. Version 2. Ann Occup Hyg. 2009;53(8):815-27.

45. Fisher EM, Shaffer RE. A method to determine the available UV-C dose for the decontamination of filtering facepiece respirators. J Appl Microbiol. 2011;110(1):287-95.

46. Lindsley WG, Martin SB Jr, Thewlis RE, Sarkisian K, Nwoko JO, Mead KR, Noti JD. Effects of Ultraviolet Germicidal Irradiation (UVGI) on N95 Respirator Filtration Performance and Structural Integrity. Version 2. J Occup Environ Hyg. 2015;12(8):509-17.

47. Rowan NJ, Laffey JG. Challenges and solutions for addressing critical shortage of supply chain for personal and protective equipment (PPE) arising from Coronavirus disease (COVID19) pandemic - Case study from the Republic of Ireland. Sci Total Environ. 2020 Jul 10;725:138532.

48. Rimmer A. Covid-19: Experts question guidance to reuse PPE. BMJ. 2020;369:m1577.

49. Lockhart SL, Duggan LV, Wax RS, Saad S, Grocott HP. Personal protective equipment (PPE) for both anesthesiologists and other airway managers: principles and practice during the COVID-19 pandemic. Can J Anaesth. 2020;67(8):1005-15.

50. Jessop ZM, Dobbs TD, Ali SR, Combellack E, Clancy R, Ibrahim N, et al. Personal Protective Equipment (PPE) for Surgeons during COVID-19 Pandemic: A Systematic Review of Availability, Usage, and Rationing. Br J Surg. 2020:10.1002/bjs.11750. 
51. Roberge RJ. Face shields for infection control: A review. Version 2. J Occup Environ Hyg. 2016;13(4):235-42.

52. Verbeek JH, Rajamaki B, Ijaz S, Sauni R, Toomey E, Blackwood B, et al. Personal protective equipment for preventing highly infectious diseases due to exposure to contaminated body fluids in healthcare staff. Cochrane Database Syst Rev. 2020;4(4):CD011621.

53. Fillingham YA, Grosso MJ, Yates AJ, Austin MS. Personal Protective Equipment: Current Best Practices for Orthopedic Teams. J Arthroplasty. 2020;35(7S):S19-S22.

54. Personal protective equipment (PPE) needs in healthcare settings for the care of patients with suspected or confirmed novel coronavirus (2019-nCoV). [Internet].ECDC, European Union. Available from: https://www.ecdc.europa.eu/sites/default/files/documents/novelcoronavirus-personal-protective-equipment-needs-healthcare-settings.pdf

55. Izzetti R, Nisi M, Gabriele M, Graziani F. COVID-19 Transmission in Dental Practice: Brief Review of Preventive Measures in Italy. J Dent Res. 2020:22034520920580.

56. Houghton C, Meskell P, Delaney H, Smalle M, Glenton C, Booth A, et al. Barriers and facilitators to healthcare workers' adherence with infection prevention and control (IPC) guidelines for respiratory infectious diseases: a rapid qualitative evidence synthesis. Cochrane Database Syst Rev. 2020;4(4):CD013582.

57. Cook TM. Personal protective equipment during the coronavirus disease (COVID) 2019 pandemic - a narrative review. Anaesthesia. 2020;75(7):920-7.

58. Heinzerling A, Stuckey MJ, Scheuer T, Xu K, Perkins KM, Resseger H, Magill S, Verani JR, Jain S, Acosta M, Epson E. Transmission of COVID-19 to Health Care Personnel During Exposures to a Hospitalized Patient - Solano County, California, February 2020. MMWR Morb Mortal Wkly Rep. 2020;69(15):472-6.

59. Chen W, Huang Y. To Protect Health Care Workers Better, To Save More Lives With COVID-19. Anesth Analg. 2020;131(1):97-101.

60. Hoe Gan W, Wah Lim J, Koh D. Preventing intra-hospital infection and transmission of COVID-19 in healthcare workers. Saf Health Work. 2020;11(2):241-3.

61. Semple S, Cherrie JW. Covid-19: Protecting Worker Health. Ann Work Expo Health. 2020;64(5):461-4.

62. Canova V, Lederer Schläpfer H, Piso RJ, Droll A, Fenner L, Hoffmann T, et al. Transmission risk of SARS-CoV-2 to healthcare workers -observational results of a primary care hospital contact tracing. Swiss Med Wkly. 2020;150:w20257. 